\title{
Tuberculous Bronchoesophageal Fistula in a Patient Infected with the HIV Virus
}

Primary or secondary esophageal tuberculosis is a rare clinical picture, accounting for about $3 \%$ of cases of digestive tract sites of the disease $(1,2)$. Tuberculous bronchoesophageal fistulas are even rarer, and to my knowledge fewer than 30 cases have been described in the English-language literature. A case of tuberculous bronchoesophageal fistula in a patient infected with the human immunodeficiency virus (HIV) is described here.

A 37-year-old HIV-positive patient was referred to our hospital for upper gastrointestinal endoscopy, with a presumptive diagnosis of esophageal candidiasis. He had been complaining of periodical night sweats for three months, with a retrosternal burning sensation and mild dysphagia in the previous two weeks, and occasional vomiting after meals. The physical examination was normal, although the patient had a wasted appearance. Laboratory tests showed an erythrocyte sedimentation rate of $80 \mathrm{~mm} / \mathrm{h}$, with a positive HIV test (enzyme-linked immunosorbent assay). The chest radiograph showed a slight widening of the upper mediastinum and calcified mediastinal adenopathy, without other abnormalities or classical hallmarks of pulmonary tuberculosis such as cavitation and apical distribution. At endoscopy, an esophageal fistula was identified at the level of the middle third of the esophagus (Figure 1). Contrast imaging showed that the fistula communicated with bronchi. This finding raised a suspicion of tuberculosis, and three consecutive sputum specimens were examined and found to be positive for acid-fast bacilli (AFB) of Mycobacterium tuberculosis. A short course of antituberculous chemotherapy resulted in the loss of the current symptoms, and led to clinical and endoscopic closure of the fistula.

This case shows that, in fistulas caused by esophageal tuberculosis, there may be neither characteristic symptoms or diagnostic signs, nor a typical chest radiograph or laboratory findings. Although the condition is rare, a tuberculous bronchoesophageal fistula should be considered in the differential diagnosis of dysphagia and retrosternal burning pain, especially in patients who are HIVseropositive, or who have sputum positive for AFB. Prompt and

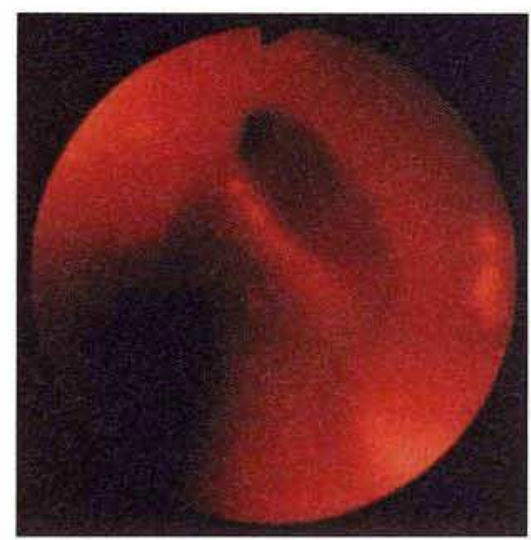

Figure 1: Bronchoesophageal fistula in the middle third of the esophagus.

correctly performed antituberculous chemotherapy leads to the healing of the fistulas, avoiding surgery in most cases and leading to resolution of the presenting symptoms.

\section{Ravera}

Gastroenterology Unit, Hoima Hospital, Kampala, Uganda

\section{References}

1. Bennani A, Dafiri N, Ouazzani L, et al. An unusual cause of dysphagia: esophageal tuberculosis. Ann Gastroenterol Hepatol (Paris) $1994 ; 30: 260-4$

2. Monig SP, Schmidt R, Wolters U, Krug B. Esophageal tuberculosis: a differential diagnostic challenge. Am J Gastroenterol 1995; 90: $153-4$.

Corresponding Author

M. Ravera, M.D., c/o AVSI

P.O. Box 6785, Kampala, Uganda 